PROCEEDINGS OF THE AMERICAN MATHEMATICAL SOCIETY

Volume 124, Number 6, June 1996

\title{
WKB-TYPE APPROXIMATIONS FOR SECOND-ORDER DIFFERENTIAL EQUATIONS IN $C^{*}$-ALGEBRAS
}

\author{
RENATO SPIGLER AND MARCO VIANELLO
}

(Communicated by Hal L. Smith)

\begin{abstract}
Rigorous asymptotic approximations of the WKB (or LiouvilleGreen) type are obtained for a basis of solutions to $y^{\prime \prime}+[\alpha+g(t)] y=0$ in the framework of $C^{*}$-algebras. Both cases $\alpha>0$ and $\alpha<0$ are included, thus generalizing the classical theory for scalar equations developed by F.W.J. Olver to matrix as well as to infinite-dimensional equations.
\end{abstract}

\section{INTRODUCTION}

In this paper we prove WKB-type (or Liouville-Green-type) approximation results for certain second-order differential equations in $C^{*}$-algebras. Such results apply to equations like

$$
y^{\prime \prime}+[\alpha+g(t)] y=0, \quad t \in(a, b),
$$

where $-\infty \leq a<b \leq+\infty, y$ and $g$ take values in a $C^{*}$-algebra, $\mathcal{B}$, with a unit element $e, g \in C^{0}((a, b) ; \mathcal{B})$, and $\alpha$ is a suitable (constant) normal element of $\mathcal{B}$. Moreover, $g$ is assumed to be "asymptotically negligible". In particular, the case " $\alpha>0$ " as well as that of " $\alpha<0$ " are included, thus generalizing the well-known results obtained by F.W.J. Olver [3, 4] for both oscillatory and non-oscillatory scalar equations. Recall that " $\alpha>0$ " means that $\alpha$ is Hermitian and its spectrum $\sigma(\alpha) \subset(0,+\infty)[5$, Ch.11].

Below, as in $[4$, Ch.6] for the classical case, precise error bounds in the asymptotic approximations for a basis of the (right) $\mathcal{B}$-module of solutions to (1) are obtained, and the double asymptotic nature with respect to the independent variable $x$ and the coefficient $\alpha$ is also shown clearly.

We emphasize that the only generalization of the WKB theory beyond the case of scalar second-order differential equations seems to be that to matrix differential equations which appeared in [6]. More recently, we obtained asymptotic results for equation (1) with $\alpha=0$ in the framework of general Banach algebras [7].

In Section 2, we state and prove our main theorems. They are based on the analysis of certain Volterra-Bochner integral equations satisfied by the error terms

Received by the editors November 16, 1994.

1991 Mathematics Subject Classification. Primary 34E20, 34G10, 46L05.

Key words and phrases. Linear abstract differential equations, WKB (Liouville-Green) approximation, $C^{*}$-algebras.

This work has been supported, in part, by the MURST Mathematical Analysis funds ("60\%" funds), the GNIM-CNR, and the GNFM-CNR. Hospitality by IMA at the University of Minnesota, Minneapolis, is happily acknowledged by R.S. 
appearing in the asymptotic representation of the basis solutions. Estimates are derived by successive approximations. In Section 3, finally, several comments are made and an example is worked out in detail.

\section{WKB THEOREMS IN $C^{*}$-ALGEBRAS}

The main results of this paper are given by Theorems 2.1 and 2.2 below. In fact, it is convenient to state separately a theorem for the case $\alpha>0$ ("oscillatory case"). In the following, $\exp \{\cdot\}$ denotes the exponential function in the complex domain as well as in Banach algebras. The symbol $o(1)$ represents any infinitesimal $\mathcal{B}$-valued function.

Theorem 2.1. Suppose equation (1) is given with $\alpha>0$, and $g \in C^{0}((a, b) ; \mathcal{B})$ such that

$$
\int_{a}^{b}\|g(t)\| d t<\infty
$$

Then, for each $\tau \in[a, b]$, the right $\mathcal{B}$-module of solutions to (1) is generated by a pair $\left(y_{1}(t), y_{2}(t)\right)$ such that

$$
y_{1}(t)=\exp \left\{i \alpha^{1 / 2} t\right\}\left[e+\varepsilon_{1}(t)\right], y_{2}(t)=\exp \left\{-i \alpha^{1 / 2} t\right\}\left[e+\varepsilon_{2}(t)\right],
$$

$\alpha^{1 / 2}$ denoting the positive square root of $\alpha$, with

$$
\left\|\varepsilon_{j}(t)\right\| \leq \exp \{V(t)\}-1,\left\|\alpha^{-1 / 2} \varepsilon_{j}^{\prime}(t)\right\| \leq \exp \{V(t)\}-1, \quad j=1,2, \quad t \in(a, b),
$$

where

$$
V(t):=\int_{t \wedge \tau}^{t \vee \tau}\left\|\alpha^{-1 / 2} g(s)\right\| d s
$$

Proof. Assume, without loss of generality, $\tau=b$. Equation (1) has a solution like $y_{1}(t)$ in (3) iff the error term, $\varepsilon_{1}(t)$, satisfies the "error equation"

$$
\varepsilon_{1}^{\prime \prime}+2 i \alpha^{1 / 2} \varepsilon_{1}^{\prime}+\exp \left\{-i \alpha^{1 / 2} t\right\} g(t) \exp \left\{i \alpha^{1 / 2} t\right\}\left(e+\varepsilon_{1}\right)=0 .
$$

In deriving equation (6) we exploited the fact that $\alpha^{1 / 2}$ and $\alpha$ commute with $\exp \left\{i \alpha^{1 / 2} t\right\}$. Note that $z:=\exp \left\{i \alpha^{1 / 2} t\right\}$ is a solution of the "unperturbed" equation $z^{\prime \prime}+\alpha z=0$. It is easily seen that every $C^{2}$-solution of the integral equation of the Volterra-Bochner type,

$$
\begin{aligned}
& \varepsilon_{1}(t)=-\frac{i}{2} \int_{t}^{b} {\left[e-\exp \left\{-2 i \alpha^{1 / 2}(t-s)\right\}\right] } \\
& \cdot \exp \left\{-i \alpha^{1 / 2} s\right\} \alpha^{-1 / 2} g(s) \exp \left\{i \alpha^{1 / 2} s\right\}\left[e+\varepsilon_{1}(s)\right] d s
\end{aligned}
$$

also solves equation (6) (cf. [7] for a similar derivation).

Define recursively the sequence

$$
\begin{aligned}
h_{j+1}(t):=-\frac{i}{2} \int_{t}^{b} & {\left[e-\exp \left\{-2 i \alpha^{1 / 2}(t-s)\right\}\right] } \\
\cdot & \exp \left\{-i \alpha^{1 / 2} s\right\} \alpha^{-1 / 2} g(s) \exp \left\{i \alpha^{1 / 2} s\right\}\left[e+h_{j}(s)\right] d s,
\end{aligned}
$$


$j=0,1,2, \ldots$, where $h_{0}(t):=0$. Such a sequence is well-defined, with $h_{j} \in$ $C^{2}((a, b) ; \mathcal{B})$ for every $j$, in view of $(2)$. This can be proved easily by induction on $j$ (cf. [7]). Then we prove that the "Liouville-Neumann" series [4]

$$
\varepsilon_{1}(t):=\sum_{j=0}^{\infty}\left[h_{j+1}(t)-h_{j}(t)\right]
$$

converges uniformly on $(a, b)$. Indeed, assuming as an inductive hypothesis that

$$
\left\|h_{j}(t)-h_{j-1}(t)\right\| \leq \frac{V^{j}(t)}{j !}
$$

$V(t)$ being defined in (5) with $\tau=b$, which is clearly true for $j=1$, we obtain

$$
\begin{gathered}
\left\|h_{j}(t)-h_{j-1}(t)\right\| \leq \int_{t}^{b}\left\|\alpha^{-1 / 2} g(s)\right\| \frac{V^{j}(s)}{j !} d s \\
=-\frac{1}{j !} \int_{t}^{b} V^{\prime}(s) V^{j}(s) d s=\frac{V^{j+1}(t)}{(j+1) !} .
\end{gathered}
$$

The uniform convergence of (9) then follows by $V(t) \leq V(a)<\infty$ (cf. (2)), and more, the first estimate in (4) is obtained. Moreover,

$$
\begin{array}{rl}
h_{j+1}^{\prime}(t)=-\frac{i}{2} \int_{t}^{b} & 2 i \alpha^{1 / 2} \exp \left\{-2 i \alpha^{1 / 2}(t-s)\right\} \\
\cdot & \exp \left\{-i \alpha^{1 / 2} s\right\} \alpha^{-1 / 2} g(s) \exp \left\{i \alpha^{1 / 2} s\right\}\left[e+h_{j}(s)\right] d s,
\end{array}
$$

from which we get

$$
\left\|\alpha^{-1 / 2}\left[h_{j+1}^{\prime}(t)-h_{j}^{\prime}(t)\right]\right\| \leq \frac{V^{j+1}(t)}{(j+1) !} .
$$

Therefore the series $\sum_{j=0}^{\infty}\left[h_{j+1}^{\prime}(t)-h_{j}^{\prime}(t)\right]$ also converges uniformly, so that $\varepsilon_{1} \in$ $C^{1}((a, b) ; \mathcal{B})$ and the second estimate in $(4)$ is obtained. As for the second derivative,

$$
h_{j+1}^{\prime \prime}(t)=-\exp \left\{-i \alpha^{1 / 2} t\right\} g(t) \exp \left\{i \alpha^{1 / 2} t\right\}\left[e+h_{j}(t)\right]
$$

$$
-2 i \int_{t}^{b} \exp \left\{-2 i \alpha^{1 / 2}(t-s)\right\} \exp \left\{-i \alpha^{1 / 2} s\right\} \alpha^{1 / 2} g(s) \exp \left\{i \alpha^{1 / 2} s\right\}\left[e+h_{j}(s)\right] d s,
$$

from which

$$
\left\|h_{j+1}^{\prime \prime}(t)-h_{j}^{\prime \prime}(t)\right\| \leq\|g(t)\| \frac{V^{j}(t)}{j !}+2\|\alpha\| \frac{V^{j+1}(t)}{(j+1) !} .
$$

This shows that $\sum_{j=0}^{\infty}\left[h_{j+1}^{\prime \prime}(t)-h_{j}^{\prime \prime}(t)\right]$ converges uniformly on compact subsets of $(a, b)$, which ensures that $\varepsilon_{1} \in C^{2}((a, b) ; \mathcal{B})$ and yields the estimate

$$
\left\|\varepsilon_{1}^{\prime \prime}(t)\right\| \leq\|g(t)\| \exp \{V(t)\}+2\|\alpha\|(\exp \{V(t)\}-1) .
$$


Finally, it is straightforward to show that $\varepsilon_{1}(t)$ given by (9) solves the integral equation (7):

$$
\begin{gathered}
\varepsilon_{1}(t)=\sum_{j=0}^{\infty}\left[h_{j+1}(t)-h_{j}(t)\right]=h_{1}(t) \\
-\frac{i}{2} \sum_{j=1}^{\infty} \int_{t}^{b}\left[e-\exp \left\{-2 i \alpha^{1 / 2}(t-s)\right\}\right] \\
\cdot \exp \left\{-i \alpha^{1 / 2} s\right\} \alpha^{-1 / 2} g(s) \exp \left\{i \alpha^{1 / 2} s\right\}\left[h_{j}(s)-h_{j-1}(s)\right] d s \\
=h_{1}(t)-\frac{i}{2} \sum_{j=1}^{\infty} \int_{t}^{b}\left[e-\exp \left\{-2 i \alpha^{1 / 2}(t-s)\right\}\right] \\
\cdot \exp \left\{-i \alpha^{1 / 2} s\right\} \alpha^{-1 / 2} g(s) \exp \left\{i \alpha^{1 / 2} s\right\} \varepsilon_{1}(s) d s .
\end{gathered}
$$

Note that exchanging the integral and series symbols in (17) is permissible by the dominated convergence theorem for Bochner integral [1, Ch.3], in view of $(2)$ and (11).

Concerning the second solution, $y_{2}(t)$, the proof proceeds along the same lines as above, with most obvious modifications. To conclude, we show that the pair $\left(y_{1}(t), y_{2}(t)\right)$ is a basis for the right $\mathcal{B}$-module of solutions to (1), which is known to be free and of rank 2 (cf. [2], e.g.). Defining, as in [7], the Wronskian matrix

$$
\mathbf{W}(t):=\left(\begin{array}{cc}
y_{1}(t) & y_{2}(t) \\
y_{1}^{\prime}(t) & y_{2}^{\prime}(t)
\end{array}\right), \mathbf{W}(t) \in M_{2}(\mathcal{B})
$$

by reduction to the first-order case [2] it turns out that $\left(y_{1}(t), y_{2}(t)\right)$ is a basis for (1) iff $\mathbf{W}(t)$ is invertible for every $t \in(a, b)$. Since it is immediately seen that $y_{1}(t), y_{2}(t)$ are linearly independent solutions to (1) (in view of their asymptotic behavior), $\mathbf{W}(t)$ is injective for every fixed $t$. Even though in case $\mathcal{B}=M_{n}(\mathbf{C})$ this would suffice to ensure the invertibility of $\mathbf{W}(t)$, this is not enough in general. In order to prove it, note that $\varepsilon_{j}(t), \varepsilon_{j}^{\prime}(t)=o(1)$ as $t \rightarrow b^{-}$imply

$$
\mathbf{W}(t)=\left(\begin{array}{cc}
\exp \left\{i \alpha^{1 / 2} t\right\} & \exp \left\{-i \alpha^{1 / 2} t\right\} \\
i \alpha^{1 / 2} \exp \left\{i \alpha^{1 / 2} t\right\} & -i \alpha^{1 / 2} \exp \left\{-i \alpha^{1 / 2} t\right\}
\end{array}\right)
$$

$$
+\left(\begin{array}{cc}
\exp \left\{i \alpha^{1 / 2} t\right\} o(1) & \exp \left\{-i \alpha^{1 / 2} t\right\} o(1) \\
i \alpha^{1 / 2} \exp \left\{i \alpha^{1 / 2} t\right\} o(1) & -i \alpha^{1 / 2} \exp \left\{-i \alpha^{1 / 2} t\right\} o(1)
\end{array}\right)=: \mathbf{W}_{0}(t)+\mathbf{W}_{1}(t)
$$

(cf. (4)), from which

$$
\mathbf{W}(t)=\mathbf{W}_{0}(t)\left[\mathbf{I}+\mathbf{W}_{0}^{-1}(t) \mathbf{W}_{1}(t)\right],
$$

$\mathbf{W}_{0}(t)$ being invertible for every $t \in(a, b)$ (in fact, its entries commute and its determinant is invertible), and $\mathbf{I}$ denoting the unit element of $M_{2}(\mathcal{B})$. The invertibility of $\mathbf{W}(t)$ in a neighborhood of $t=b$ (and hence everywhere) follows from the 
estimate

$$
\left|\left\|\mathbf{W}_{0}^{-1}(t) \mathbf{W}_{1}(t)\right\|\right|<1,
$$

valid in a neighborhood of $b$, the triple-bar norm in $M_{2}(\mathcal{B})$ being that induced canonically by $\|\cdot\|($ cf. [7]). Inequality (21) can be promptly estabilished writing out

$$
\mathbf{W}_{0}^{-1}(t) \mathbf{W}_{1}(t)=\frac{i}{2} \alpha^{-1 / 2}\left(\begin{array}{cc}
o(1) & \exp \left\{-2 i \alpha^{1 / 2} t\right\} o(1) \\
\exp \left\{2 i \alpha^{1 / 2} t\right\} o(1) & o(1)
\end{array}\right),
$$

which is infinitesimal in $\left(M_{2}(\mathcal{B}),|\|\cdot\||\right)$, as $\left\|\exp \left\{ \pm 2 i \alpha^{1 / 2} t\right\}\right\|=1$.

Theorem 2.2. Suppose equation (1) is given with $\alpha=\beta^{2}, \sigma(\beta) \subset\{\lambda \in \mathbf{C}$ : $\operatorname{Im} \lambda \geq 0\}$, but $\exists \lambda \in \sigma(\beta)$ such that $\operatorname{Im} \lambda>0, \beta$ being an invertible normal element of $\mathcal{B}$. Assume that $g \in C^{0}((a, b) ; \mathcal{B})$ is such that

$$
\int_{a}^{b}\|\exp \{-i \beta t\} g(t) \exp \{i \beta t\}\| d t<\infty \text {. }
$$

Then there exists a solution to (1),

$$
u(t)=\exp \{i \beta t\}[e+\varepsilon(t)],
$$

with

$$
\begin{gathered}
\|\varepsilon(t)\| \leq \exp \{V(t)\}-1,\left\|\beta^{-1} \varepsilon^{\prime}(t)\right\| \leq \exp \{V(t)\}-1, \\
V(t):=\int_{t}^{b}\left\|\beta^{-1} \exp \{-i \beta s\} g(s) \exp \{i \beta s\}\right\| d t .
\end{gathered}
$$

On the other hand, assuming that

$$
\int_{a}^{b}\|\exp \{i \beta t\} g(t) \exp \{-i \beta t\}\| d t<\infty
$$

then there is a solution,

with

$$
v(t)=\exp \{-i \beta t\}[e+\eta(t)]
$$

$$
\begin{gathered}
\|\eta(t)\| \leq \exp \{W(t)\}-1,\left\|\beta^{-1} \eta^{\prime}(t)\right\| \leq \exp \{W(t)\}-1, \\
W(t):=\int_{a}^{t}\left\|\beta^{-1} \exp \{i \beta s\} g(s) \exp \{-i \beta s\}\right\| d s .
\end{gathered}
$$

Moreover, when (23) and (26) both hold, and $\operatorname{Im} \lambda>0$ for all $\lambda \in \sigma(\beta)$ : If $b=$ $+\infty$ and $W(+\infty)<\log 2$, then the right $\mathcal{B}$-module of solutions to (1) is generated by the pair $(u(t), z(t))$, with

$$
z(t)=\exp \{-i \beta t\}[e+o(1)], \quad z^{\prime}(t)=-i \beta \exp \{-i \beta t\}[e+o(1)], \text { as } t \rightarrow+\infty ;
$$

if $a=-\infty$ and $V(-\infty)<\log 2$, the right $\mathcal{B}$-module of solutions to (1) is generated by the pair $(w(t), v(t))$, with

(30) $w(t)=\exp \{i \beta t\}[e+o(1)], w^{\prime}(t)=i \beta \exp \{i \beta t\}[e+o(1)]$, as $t \rightarrow-\infty$.

Before proving Theorem 2.2, some observations are in order. 
Remark 2.3. If $W(+\infty) \geq \log 2$, one can merely restrict $(a, b)$ to $\left(a_{1}, b\right)$, with $a<a_{1}<b$, to obtain $\int_{a_{1}}^{+\infty}\|\exp \{i \beta t\} g(t) \exp \{-i \beta t\}\| d t<\log 2$. However, the second solution, $z(t)$, exhibiting the behavior in (27) will be different. Similar considerations apply when $V(-\infty) \geq \log 2$.

Remark 2.4. When $\alpha$ and $\beta$ are as in Theorem 2.2, but $\sigma(\beta) \subset \mathbf{R}$, then the hypotheses of Theorem 2.2 coincide with those of Theorem 2.1. In fact, $\beta$ is Hermitian by the spectral theorem [5] in this case, and hence $\alpha>0$. On the other hand, condition (23) (or [26]) is equivalent to (2), since $\|\exp \{ \pm i \beta t\}\|=1$ (actually, $\|\exp \{ \pm i \beta t\} g(t) \exp \{\mp i \beta t\}\| \equiv\|g(t)\|)$. It is worthwhile, at this point, to note that condition (23) [or (26)] and (2) are also equivalent when: (i) $(a, b)$ is a bounded interval; (ii) $(a, b)$ is unbounded, and $\beta$ and $g(t)$ commute for all $t$ in a neighborhood of $+\infty$ (or $-\infty$ ); (iii) $\sigma(\beta)$ is a singleton. We stress that, in general, (23) and (26) are not equivalent.

Remark 2.5. When $\alpha$ is a normal (invertible) matrix, possessing at least one nonreal eigenvalue, then there exists a square root, $\beta$, which is normal (invertible), and with non-real spectrum in the (closed) upper half-plane. In fact,

$$
\alpha=P^{-1} \operatorname{diag}\left(\lambda_{1}, \ldots, \lambda_{n}\right) P
$$

in this case, $P$ being unitary. Therefore, we can choose

$$
\beta=P^{-1} \operatorname{diag}\left(\lambda_{1}^{1 / 2}, \ldots, \lambda_{n}^{1 / 2}\right) P,
$$

with $\operatorname{Im} \lambda_{j}^{1 / 2} \geq 0$ for each $j$, since we can select the appropriate branch of the square root for each $j$.

In a general $C^{*}$-algebra, the spectral theorem ensures that a square root, $\beta$, with the required properties does exist whenever $\alpha$ is a normal (invertible) element with non-real spectrum, completely contained in the (closed) upper or lower half-plane.

Proof of Theorem 2.2. The proof of (24)-(26) can be obtained following closely the steps in Theorem 2.1. Indeed, focusing on the representation for $u(t)$ in $(24)$, exactly the same error equation in (6) as well as the integral equation in (7) are obtained for $\varepsilon(t)$ (with $\beta$ replacing $\alpha^{1 / 2}$ ). In estimating $\varepsilon(t)$ (and its derivatives) by successive approximations, the stronger condition (23) is now needed (rather than (2)). Again, all results concerning the second solution, $v(t)$, around $t=a$ are obtained similarly.

Hereafter we assume that (23) and (26) both hold true. It is not clear, however, whether $u(t)$ and $v(t)$ are linearly independent solutions to (1) (and thus, a fortiori, whether they generate the right $\mathcal{B}$-module of solutions). When $b=+\infty$ and $\operatorname{Im} \lambda>$ 0 for all $\lambda \in \sigma(\beta)$, following Olver's classical approach in [4, Thm.3.1, Ch.6] for the exponential case, we can show that the error term $\eta(t)$ in (27) has a limit and that $\eta^{\prime}(t)=o(1)$ as $t \rightarrow+\infty$. For every $\delta>0$, choosing $a_{1}=a_{1}(\delta) \in(a,+\infty)$ such that

$$
\int_{a_{1}}^{+\infty}\|\exp \{i \beta s\} g(s) \exp \{-i \beta s\}\| d t<\delta
$$

we obtain first, after standard calculations,

$$
\begin{gathered}
\left\|\eta^{\prime}(t)\right\| \leq \exp \left\{-2 m\left(t-a_{1}\right)\right\}\left(\exp \left\{W\left(a_{1}\right)\right\}-1\right)+\delta \exp \{W(+\infty)\}, \\
m:=\min _{\lambda \in \sigma(\beta)} \operatorname{Im} \lambda .
\end{gathered}
$$


Hence, $\eta^{\prime}(t)=o(1)$ as $t \rightarrow+\infty$, being $m>0$ and $\delta$ arbitrary. Finally, writing $\eta(t)+\frac{i}{2} \beta^{-2} \eta^{\prime}(t)$ using the Liouville-Neumann expansions of $\eta(t)$ and $\eta^{\prime}(t)$ [4], we obtain

$$
\|\eta(t)-\eta(s)\| \leq \frac{\left\|\beta^{-1}\right\|}{2} \exp \{W(t)\}|W(t)-W(s)|+\frac{\left\|\beta^{-2}\right\|}{2}\left(\left\|\eta^{\prime}(t)\right\|+\left\|\eta^{\prime}(s)\right\|\right),
$$

and thus, letting $t, s \rightarrow+\infty$ (independently), we see that $\eta(t)$ has a finite limit, say $\eta(+\infty)$. Therefore, from (28), since $\|\eta(+\infty)\| \leq \exp \{W(+\infty)\}-1<1$ by condition $W(+\infty)<\log 2$, we derive

$$
\begin{gathered}
z(t):=v(t)[e+\eta(+\infty)]^{-1}=\exp \{-i \beta t\}[e+o(1)], \\
z^{\prime}(t)=v^{\prime}(t)[e+\eta(+\infty)]^{-1}=-i \beta \exp \{-i \beta t\}[e+o(1)], \text { as } t \rightarrow+\infty .
\end{gathered}
$$

We are only left with the proof that the pair $(u(t), z(t))$ generates the $\mathcal{B}$-module of solutions, i.e. that the Wronskian matrix $\mathbf{W}(t)$ (cf. (18)) is invertible at every $t$. Other than in the proof of Theorem 2.1, we need the multiplicative splitting

$$
\mathbf{W}(t)=\left(\begin{array}{cc}
\exp \{i \beta t\}[e+o(1)] & \exp \{-i \beta t\}[e+o(1)] \\
i \beta \exp \{i \beta t\}[e+o(1)] & -i \beta \exp \{-i \beta t\}[e+o(1)]
\end{array}\right)
$$

$$
=\left(\begin{array}{cc}
\exp \{-i \beta t\} & 0 \\
0 & \exp \{-i \beta t\}
\end{array}\right)\left(\begin{array}{cc}
\exp \{2 i \beta t\}[e+o(1)] & e+o(1) \\
\exp \{2 i \beta t\}[i \beta+o(1)] & -i \beta+o(1)
\end{array}\right)
$$

which shows that $\mathbf{W}(t)$ is invertible (about $t=+\infty$ and hence everywhere) iff such is the second factor on the r.h.s. of (35). This is true because such a factor can be split into the sum of an invertible element and an infinitesimal element of $M_{2}(\mathcal{B})$, as in the proof of Theorem 2.1. The case $a=-\infty$ (cf. (30)) can be treated in a similar way.

The "purely exponential" case $\alpha<0$, included in Theorem 2.2, deserves a separate statement, since it corresponds to the analogue scalar case [4, Thm.2.1, Ch.6], and, at the same time, slightly sharper estimates are obtained:

Theorem 2.6. Suppose equation (1) is given with $\alpha<0$, and (23) [or (26)] is satisfied with $\beta=i(-\alpha)^{1 / 2}$. Then, all conclusions of Theorem 2.2 hold true with the only modification that $V(t) / 2[$ or $W(t) / 2]$ replaces $V(t)[$ or $W(t)]$ in the estimate (25) [or (28)].

Proof. The only thing to observe is that, in (7), $\left\|e-\exp \left\{-2 i(-\alpha)^{1 / 2}(t-s)\right\}\right\| \leq$ $1(t \geq s)$ in this case. In fact, denoting by $\lambda_{\beta}$ the elements of the spectrum of $\beta$, those of the normal element $e-\exp \left\{-2 i(-\alpha)^{1 / 2}(t-s)\right\}$ are given by $1-$ $\exp \left\{-2 \lambda_{\beta}(t-s)\right\} \leq 1(t \geq s)$.

Remark 2.7. Under the additional assumptions that $\alpha$ and $g(t)$ commute for all $t$ in a neighborhood of $+\infty$, the existence of a solution, $z(t)$, with the asymptotic behavior in (29) can be proved by a straightforward application of an abstract version of L'Hôpital's theorem [9]; see also [8]. Indeed, in such case, $z(t)$ can be represented as $z(t)=u(t) \int_{t}^{+\infty} u^{-2}(s) d s$, and $u(t)$ commutes with $\beta$ (which fact can be proved via the series representation of the error term, $\varepsilon(t))$. Hence, 
$\exp \{i \beta t\} z(t)=[e+\varepsilon(t)] \exp \{2 i \beta t\} \int_{t}^{+\infty} u^{-2}(s) d s \sim \exp \{2 i \beta t\} \int_{t}^{+\infty} u^{-2}(s) d s=$ : $g^{-1}(t) f(t)$ with $g(t)=\exp \{-2 i \beta t\}$. By L'Hôpital's theorem proved in [9], it then follows that $\lim _{t \rightarrow+\infty} \exp \{i \beta t\} z(t)=\lim _{t \rightarrow+\infty}\left(g^{\prime}(t)\right)^{-1} f^{\prime}(t)=e$; cf. [9, §3.1]. The asymptotic behavior of $z^{\prime}(t)$ as $t \rightarrow+\infty$ can also be obtained by a similar application of L'Hôpital's rule.

\section{Comments And EXAmples}

The following remarks generalize to the abstract case certain properties of the Liouville-Green approximation for the scalar case; cf. [4, Ch.6].

Remark 3.1. When $g(t)$ is Hermitian, in Theorem 2.1 the basis solutions, $y_{1}(t)$, $y_{2}(t)$, are "conjugate" [5, Thm.11.15], while in Theorem 2.2 , if $\beta$ is "purely imaginary", i.e. $\beta=i \gamma$ with $\gamma$ Hermitian, then $u(t)$ and $v(t)$ are Hermitian. In the latter case, when $\gamma$ is positive $z(t)$ and $w(t)$ are also Hermitian (cf. Theorem 2.6). All these facts can be ascertained by using the series representations of the error terms along with the continuity of involution.

Remark 3.2. The typical feature of the classical Liouville-Green approximation of exhibiting a "double asymptotic nature" with respect to the independent variable and the parameter $\alpha$ persists in the abstract case. In fact, (5), (25), and (28) show that the error terms are of order $O\left(\left\|\alpha^{-1 / 2}\right\|\right)$. Note that $\alpha^{-1 / 2} \rightarrow 0$ iff $\alpha^{-1} \rightarrow 0$. In fact, by the spectral theorem, $\left\|\alpha^{-1 / 2}\right\|=\rho\left(\alpha^{-1 / 2}\right)=\left(\rho\left(\alpha^{-1}\right)\right)^{1 / 2}=\left\|\alpha^{-1}\right\|^{1 / 2}$.

A further observation is displayed in the following

Remark 3.3. In Theorem 2.2, the basis solutions $u(t)$ and $z(t)$ (or $v(t)$ and $w(t)$ ) can be termed recessive and dominant, respectively, as in the scalar case. In fact, it is natural to define "recessive" every solution $y_{r}(t)$ of $(1)$ such that another solution $y_{d}(t)$ exists with $y_{d}^{-1}(t) y_{r}(t) \rightarrow 0$ as $t \rightarrow+\infty$ (and $y_{d}(t)$ is termed "dominant"). Now, it is easy to show that the subset of all recessive solutions is a cyclic submodule (of the right $\mathcal{B}$-module of solutions of (1)). In fact, suppose that $y_{r}(t)$ is any given recessive solution of (1) and $y_{r}=u c_{1}+z c_{2}, y_{d}=u c_{3}+z c_{4}, c_{i} \in \mathcal{B}$. Then, $z^{-1} u c_{1}+c_{2}=\left(z^{-1} u c_{3}+c_{4}\right) y_{d}^{-1} y_{r} \rightarrow 0$ as $t \rightarrow+\infty$, and hence $c_{2}=0$. It follows that $u(t)$ is the only solution of (1) possessing the asymptotic behavior in (24).

New features of the Liouville-Green approximation appear in the higher-dimensional case with respect to the classical scalar case. Indeed, we have the following

Remark 3.4. In the non-commutative case, Theorem 2.2 may be applicable also with $g(t)$ non-integrable, e.g. even when $g(t)$ is of exponential order. Take for instance $\mathcal{B}=M_{2}(\mathbf{C}),(a, b)=(1,+\infty)$, and

$$
\alpha:=\left(\begin{array}{cc}
\lambda_{1}^{2} & 0 \\
0 & \lambda_{2}^{2}
\end{array}\right), \operatorname{Im} \lambda_{2}>\operatorname{Im} \lambda_{1} \geq 0, g(t):=\left(\begin{array}{cc}
0 & h(t) \\
0 & 0
\end{array}\right),
$$

with $h(t)=O(\exp \{\mu t\}), \mu<\operatorname{Im} \lambda_{2}-\operatorname{Im} \lambda_{1}$. Then, $\exp \{-i \beta t\} g(t) \exp \{i \beta t\}$ is integrable on $(1,+\infty)$ since its only non-zero entry is $h(t) \exp \left\{i\left(\lambda_{2}-\lambda_{1}\right) t\right\}$; cf. (23).

The following observation will be used in the concluding example: 
Remark 3.5. From (3) in Theorem 2.1, obtains

$$
y_{1}^{\prime}(t)=i \alpha^{1 / 2} \exp \left\{i \alpha^{1 / 2} t\right\}[e+\xi(t)],
$$

with $\xi(t)=\varepsilon_{1}(t)-i \alpha^{-1 / 2} \varepsilon_{1}^{\prime}(t)$. Hence $\|\xi(t)\| \leq 2[\exp \{V(t)\}-1]$; cf. (4). Similarly one can estimate the error term in the asymptotic representation of $y_{2}^{\prime}(t)$, and also in Theorem 2.2 as for $u^{\prime}(t), v^{\prime}(t)$.

Example 3.6. Consider, in (1), $g(t)=O\left(t^{-p}\right), p>1,(a, b)=(1,+\infty)$, and $\alpha>0$. Then

$$
\begin{gathered}
y_{1,2}(t)=\exp \left\{ \pm i \alpha^{1 / 2} t\right\}\left[e+O\left(\left\|\alpha^{-1 / 2}\right\| \frac{t^{1-p}}{p-1}\right)\right], \\
y_{1,2}^{\prime}(t)= \pm i \alpha^{1 / 2} \exp \left\{ \pm i \alpha^{1 / 2} t\right\}\left[e+O\left(\left\|\alpha^{-1 / 2}\right\| \frac{t^{1-p}}{p-1}\right)\right]
\end{gathered}
$$

see Remark 3.5. Note that, in this case, the double asymptotic nature of the Liouville-Green approximation manifests also with respect to the parameter $p$ (as $p \rightarrow+\infty)$. Also, it is worth emphasizing that the constants implied by the $O$ symbols in (38) can be immediately obtained from that in $g(t)=O\left(t^{-p}\right)$, thus allowing for a precise evaluation of the error bounds in (38). This fact can be useful for numerical purposes, for instance in the matrix case, where $\left\|\alpha^{-1 / 2}\right\|$ can be explicitly estimated computing the eigenvalues of $\alpha$.

\section{REFERENCES}

1. E. Hille and R.S. Phillips, Functional Analysis and Semi-groups, Amer. Math. Soc. Coll. Publ., vol. 31, Amer. Math. Soc., Providence, 1957. MR 19:664d

2. E. Hille, Lectures on ordinary differential equations, Addison-Wesley, Reading, 1969. MR 40:2939

3. F.W.J. Olver, Error bounds for the Liouville-Green (or WKB) approximation, Proc. Cambridge Philos. Soc. 57 (1961), 790-810. MR 24:A313

4. F.W.J. Olver, Asymptotics and Special Functions, Academic Press, New York, 1974. MR 55:8655

5. W. Rudin, Functional Analysis, McGraw-Hill, New York, 1973. MR 51:1315

6. D.R. Smith, Decoupling of recessive and nonrecessive solutions for a second-order system, J. Differential Equations 68 (1987), 383-399. MR 88i:34081

7. R. Spigler and M. Vianello, Finite moments perturbations of $y^{\prime \prime}=0$ in Banach algebras, Proc. Amer. Math. Soc. 119 (1993), 97-103. MR 93k:34144

8. R. Spigler and M. Vianello, Extending L'Hôpital's theorem to B-modules, J. Math. Anal. Appl. 179 (1993), 638-645. MR 94j:46050a

9. M. Vianello, A generalization of L'Hôpital's rule via absolute continuity and Banach modules, Real Anal. Exchange 18 (1993), 557-567. MR 94c:26028

Dipartimento di Matematica, Università di Lecce, Via Provinciale Lecce-Arnesano, P.O. Box 193, 73100, LeCCE, ItALY

E-mail address: spigler@ingle01.unile.it

Dipartimento di Matematica Pura e Applicata, Università di Padova, Via Belzoni 7 , 35131, Padova, Italy

E-mail address: marcov@euler.math.unipd.it 